\title{
Obtención de espectros usando un smartphone en la clase de Física ${ }^{+*}$
}

\author{
Esteban Guillermo Szigety ${ }^{1}$ \\ Luis Jaime Bernal ${ }^{1}$ \\ Luis Bilbao ${ }^{1}$ \\ Gabriel Horacio Pérez ${ }^{l}$ \\ Departamento de Física - Universidad Nacional de Mar del Plata \\ Buenos Aires - Argentina
}

\section{Resumen}

Este artículo presenta los fundamentos para convertir un teléfono inteligente (o smartphone) en un espectrómetro casero. Utilizando un Compact Disc Digital Audio y una ranura ubicada adecuadamente se puede observar, entre otros, el espectro de emisión de una lámpara fluorescente compacta. Además, en este trabajo se incluyen los pasos metodológicos para medir las lineas de Fraunhofer del Sol de mayor ancho espectral. La propuesta está pensada para llevar adelante una clase práctica de espectros lumínicos tanto en instituciones de educación media como así también en cursos introductorios de física universitaria.

Palavras-chave: Espectroscopio; Trabajo Práctico de Laboratorio; Red de Difracción; Smartphone; Líneas de Fraunhofer.

\begin{abstract}
This article presents the basics for converting a smartphone as a home spectrometer. Using a Compact Disc Digital Audio and a properly located slot, the emission spectrum of a compact fluorescent lamp and other sources can be observed. This work includes the methodological steps to measure the Fraunhofer's lines of the sun with the greatest spectral width. The proposal is designed to carry out a practical class of
\end{abstract}

\footnotetext{
${ }^{+}$Obtaining spectra using a smartphone in Physics class

* Recebido: agosto de 2019. Aceito: novembro de 2019.

${ }^{1}$ E-mails: esteszige@gmail.com; bernal314@gmail.com; bilbaolucho@yahoo.com; ghperez@mdp.edu.ar
} 
light spectra both from institutions of secondary education as well as in introductory courses in university physics.

Keywords: Spectroscope; Practical Laboratory Work; Diffraction Grating; Smartphone; Fraunhofer Lines.

\section{Introducción}

El uso de Compact Disc Digital Audio (CD) para obtener espectros visibles en la enseñanza de física y química ha sido ampliamente difundido desde la década del noventa hasta la fecha. La accesibilidad a dicho recurso y la propiedad de su superficie para descomponer la luz lo convierte en un insumo accesible y de bajo costo para realizar actividades en el aula. Los artículos en revistas de educación en ciencias donde se puede encontrar las diversas aplicaciones educativas realizadas con CD y DVD son, entre otras: Kalinowski y Dias Garcia (1990), Kruglyak (1991), Wakabayashi, Hamada y Sone (1998), Catelli (1999), Zanetti y Harris (1993) y Knauer (2002). Esta breve enumeración permite comprender la importancia que se le dio a estos objetos cotidianos dentro de la enseñanza de la física y la química. Antes de la aparición del CD la posibilidad de tener una red de difracción estaba limitada a conseguir en el mercado productos especializados para el laboratorio de óptica. También se propuso usar intensas fuentes de luz, como retroproyectores para permitir visualizar el espectro continuo o discontinuo sobre una pantalla (BURMAN, 1991; SADLER,1991). Hoy en día existen dispositivos superadores para fines didácticos como el RSpec Explorer (LINCOLN, 2018; HOWE; SITAR, 2014) que ha mostrado una gran versatilidad de usos permitiendo que se obtengan espectros en tiempo real y digitalizados.

La obtención de los espectros lumínicos de distintas fuentes le provee al docente una herramienta útil para abordar la problemática que presentan los estudiantes para comprender los conceptos de luz y color (SADLER, 1991). Basados en las investigaciones de Sadler podemos afirmar que la actividad experimental sobre generación y visualización de espectros de distintas fuentes ayuda a que los estudiantes alcancen una compresión correcta sobre la descomposición y naturaleza de la luz.

El presente trabajo hace uso de teléfonos móviles o smartphone con que los estudiantes secundarios y universitarios asisten a clase, convirtiéndolo en un instrumento de medición para analizar líneas espectrales, mediante la adición de un fragmento de CD.

\section{II. ¿Cómo se ve el mundo a través de una red de difracción?}

Existen redes de difracción por reflexión o por transmisión. Si tomamos un CD y reflejamos un haz luminoso, veremos que su superficie espejada nos ofrece una serie de destellos coloreados. En este caso el disco se está comportando como una red por reflexión. Para obtener una red por transmisión de un CD deberemos extraer la delgada capa o cobertura 
que sirve para identificar el disco. Este ingenioso procedimiento fue idea de Catelli, Giovannini y Oliveira (2017) quien llama este procedimiento "depilar un disco". Si bien Knauer (2002) propone usar la cubierta protectora que viene en la compra de cilindros de 100 o $50 \mathrm{CD}$, actualmente los $\mathrm{CD}$ se encuentran en desuso, por lo que es más factible y económico utilizar un CD obsoleto al que se pueda aplicar la técnica de "depilar".

La técnica de "depilar" requiere de una cinta adhesiva de buena calidad; se sugiere del tipo de embalar que son anchas y muy adherentes. Se pega la cinta sobre la cara que se escribe o se encuentra la marca del CD. Es posible mejorar el contacto repasando con el dedo o un bolígrafo. Luego se retira la cinta desde el borde del disco y se observa que la capa protectora se despega para dejar expuesta las ranuras microscópicas sobre el cual se labran los datos. Con alcohol etílico de uso medicinal es posible extraer los restos de pegamento de la cinta en caso que hayan quedado, sin dañar el disco.

Bajo estas condiciones, el CD “depilado" se comporta como una red por transmisión. A continuación se procede a recortar un octavo del total, en forma de sector circular y se ubica delante de la lente la cámara del smartphone (ver Fig. 1). Teniendo en cuenta que la curvatura de las ranuras es mayor cerca del centro del disco se sugiere que lente de la cámara este próxima al borde exterior, debido que allí las ranura presentar un radio de curvatura mayor y por lo tanto se producirá una mejor calidad de haz difractado.

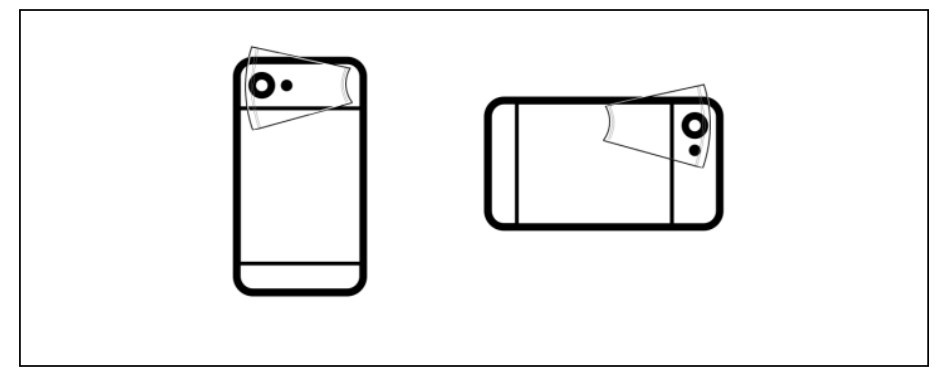

Fig. 1 - Formas de ubicar el sector circular del CD sobre la parte posterior del smartphone. Luego con una cinta adhesiva se puede terminar de fijar.

La red de transmisión puesta delante de la cámara (parte posterior del smartphone) producirá, a los costados de las fuentes lumínicas que se fotografíen, un espectro visible. Aún el dispositivo no está preparado para analizar el espectro de una lámpara o del Sol, pero será posible obtener llamativas fotografías de fuentes luminosas, objetos, lámparas de todo tipo, etc. Frecuentemente para hacer que el espectro se vea nítido en la cámara el enfoque debe ser dirigido al objeto y no al espectro.

El espectro que se aprecia en las imágenes fotografiadas obtenidas es producto de la interferencia constructiva de la luz al pasar por las ranuras del CD. Estos patrones de interferencia se los denomina máximos de difracción y su ubicación angular está dada por la longitud de onda de la luz según la siguiente expresión: 


$$
a\left(\operatorname{sen} \theta_{m}-\operatorname{sen} \theta_{i}\right)=m \lambda
$$

Donde $a$ es el espaciado de las ranuras de la red, $\lambda$ la longitud de onda incidente, $\theta_{i} \mathrm{el}$ ángulo incidente y $\theta_{m}$ el ángulo difractado de orden $m$.

La Fig. 2 muestra un haz monocromático (una sola longitud de onda) incidiendo sobre una red, como podría ser el caso del CD. Los máximos de difracción son varios, y se los numera con órdenes $(m=0,1 \mathrm{y}-1, \ldots)$. Los ángulos $\theta_{i}$ y $\theta_{m}$ son medidos desde la normal. En esta experiencia el fragmento "depilado" de CD en conjunto con la cámara del teléfono permite observar cómodamente los órdenes 1 y -1 . Órdenes superiores quedan fuera del ángulo de observación de la cámara.

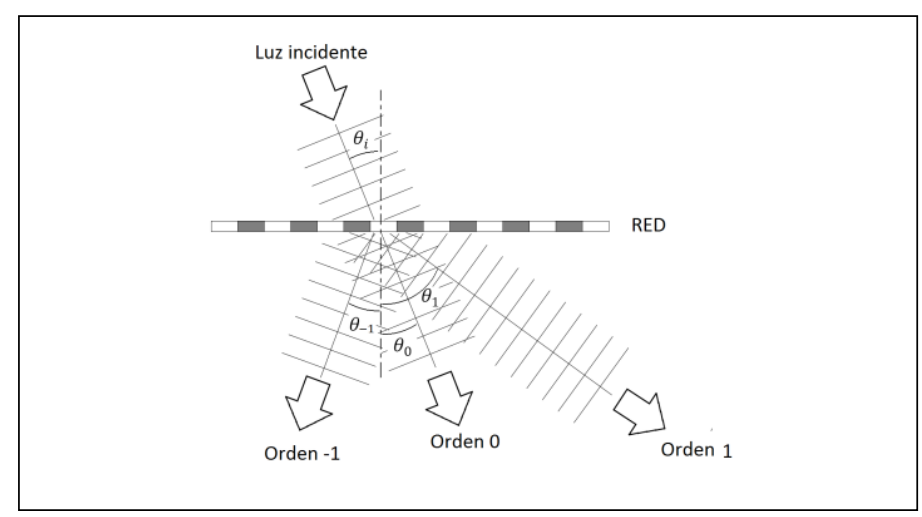

Fig. 2 - Red difractando un rayo incidente en varios rayos de distintos órdenes.

La longitud de onda de los haces que conforman el espectro son determinantes para definir el ángulo difractado $\theta_{m}$. Analizando una fuente monocromática a través este espectrómetro se visualiza copias de la fuente en posiciones angulares simétricas como se observa en la figura 3. La red está generando para cada punto de la fuente un máximo de difracción que le corresponde para esa longitud de onda. El máximo de orden cero no se desvía y por lo tanto la fuente original se observa en la posición central entre medio del orden $1 \mathrm{y}-1$. Cuando la fuente tiene una luz compuesta con varias longitudes de onda discretas observamos varias copias de la fuente ubicadas en distintas posiciones (ver Fig. 3) y el máximo de orden cero es la imagen de la fuente original. Una explicación más detallada de estas imágenes se puede encontrar también en el trabajo de Catelli (2006) donde aparecen fotografías de fuentes extensas emitiendo longitudes de onda discontinuas.

En la Fig. 3 todos los espectros fueron obtenidos usando el espectrómetro propuesto; la fuente lumínica - en forma de hombrecillo bailando - fue diseñada con el programa Microsoft Paint ${ }^{\odot}$, luego exportada a una diapositiva de Microsoft PowerPoint $^{\odot}$ y proyectada en pantalla completa en el monitor de una laptop. Las imágenes de la Fig. 3 se obtuvieron cambiando el color del hombrecillo. 


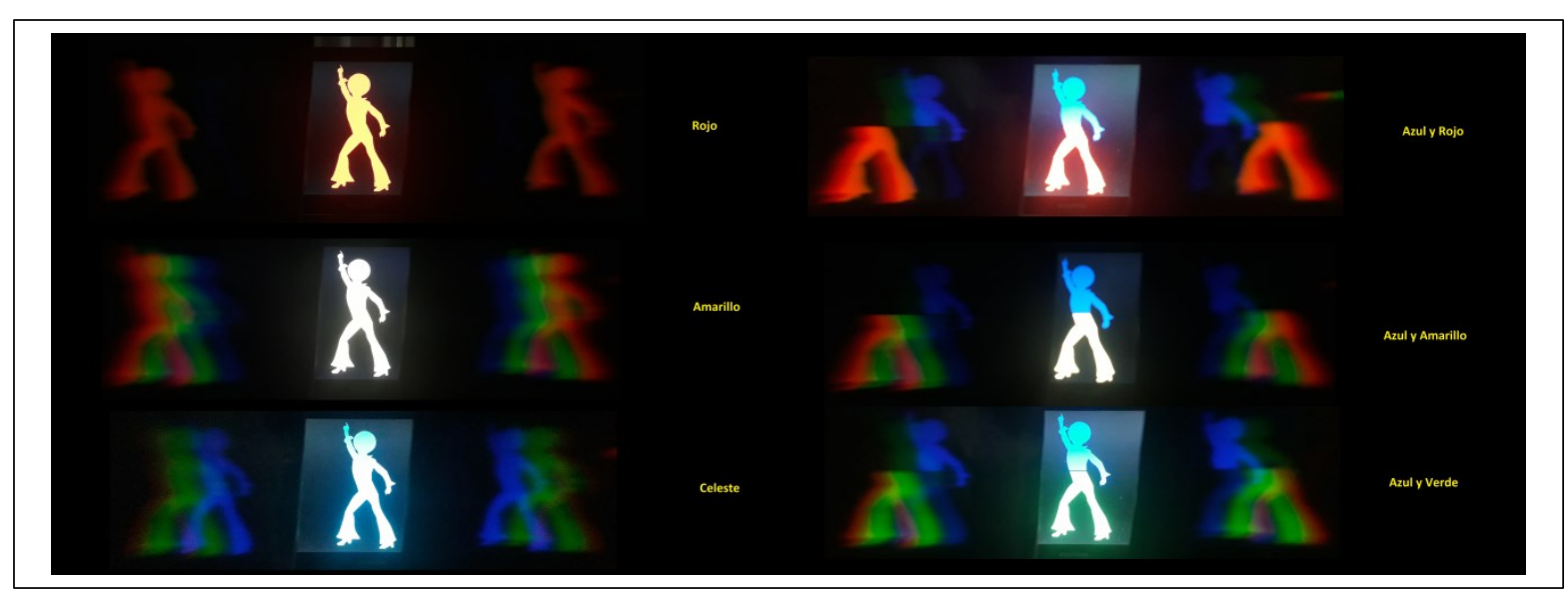

Fig. 3 - Un hombrecillo generado por la pantalla de la laptop con distintos colores. Dados que el contraste de la intensidad lumínica entre el hombrecillo central (orden cero) y la descomposición producida por la red (orden 1 y -1) es muy grande, el color del hombrecillo central ha quedado saturado y la cámara no reproduce el color original, debido a esto al costado figura se menciona su color. En la segunda columna su cuerpo del hombrecillo es bicolor.

Como se observa hasta aquí, el CD genera tantas copias de la fuente lumínica como frecuencias contenga. Si la fuente emite o refleja luz continua se observará una repetición infinita de la fuente en la imagen virtual. Esto no se consigue en la Fig. 3 debido a que el sistema de generación de imágenes de una pantalla de laptop es a través del sistema $R G B$ (en inglés: red, green y blue) y por lo tanto independientemente de color utilizado siempre se observara una suma aditiva de las tres frecuencias.

Cabe detallar que el tamaño angular del objeto es un factor fundamental si se desea que no se superpongan las distintas imágenes como claramente ocurre en la figura 3. El tamaño angular de la fuente es determinante cuando las longitudes de onda que se quieren observar se encuentran en un rango muy estrecho. Por lo tanto es importante definir el tamaño angular o ancho angular de la fuente por medio de la siguiente expresión:

$$
\Delta \theta \cong \frac{d}{L}
$$

Donde $\Delta \theta$ es el tamaño angular en radianes con que observamos el objeto, $d$ es el tamaño del objeto y $L$ la distancia a la que nos encontramos del mismo. Se puede apreciar en (2) que una forma de obtener un ancho angular menor es reduciendo el tamaño $d$ del objeto. Pero también podríamos aumentar $L$, y alcanzar el mismo resultado. La expresión (2) es aproximada debido a que mayormente $\Delta \theta$ un ángulo pequeño o también que $d \ll L$.

El espectrómetro que se presenta aquí está pensado para observar espectros de distintas fuentes, particularmente para analizar los espectros discontinuos generados por los tubos fluorescentes, lámparas fluorescentes compactas (LFC). En estos espectros ocurre que 
algunas líneas se encuentran muy próximas y se hace necesario verificar que la red sea capaz de diferenciarlas.

En el ámbito de la espectrometría esto se denomina resolver dos líneas espectrales. Un clásico ejemplo lo constituyen las lámparas de mercurio de alta presión que presentan el conocido "doblete del mercurio". Estas longitudes de onda son extremadamente próximas (577 y $579 \mathrm{~nm}$ ). La forma más sencilla para poder observarlas como dos líneas separadas consiste en anteponer a la lámpara una rendija que reduzca el tamaño angular de la fuente para resolver ambas líneas.

Se sugiere realizar la siguiente actividad para profundizar el concepto de resolución espectral. Se dibuja un cuadrado de bordes blancos con fondo oscuro en el centro de una diapositiva del programa PowerPoint ${ }^{\odot}$. Luego se construyen nuevas diapositivas en las que la imagen mantiene la altura, pero no así su ancho que irá disminuyendo hasta que la figura se convierta en una línea. En un cuarto oscuro se proyecta la secuencia de diapositivas a pantalla completa en el monitor de la laptop y se toman varias fotografías con el dispositivo propuesto conservando la distancia $(L)$ entre la cámara y la pantalla. La Fig. 4 ilustra el resultado de la actividad (vale aclarar que la red se corrió levemente de su eje de simetría para que las imágenes virtuales se puedan observar superpuestas y luego a medida que angostamos el rectángulo comenzamos a definir líneas). El resultado que se puede apreciar claramente es que las longitudes de onda utiliza por la laptop responden al sistema $R G B$ para producir la luz blanca. Se puede detectar también, que el ancho espectral del color verde en el sistema $R G B$ de la laptop es mayor que el del azul o el rojo. Análisis similares se pueden realizar con otros dispositivos o iluminación LED.

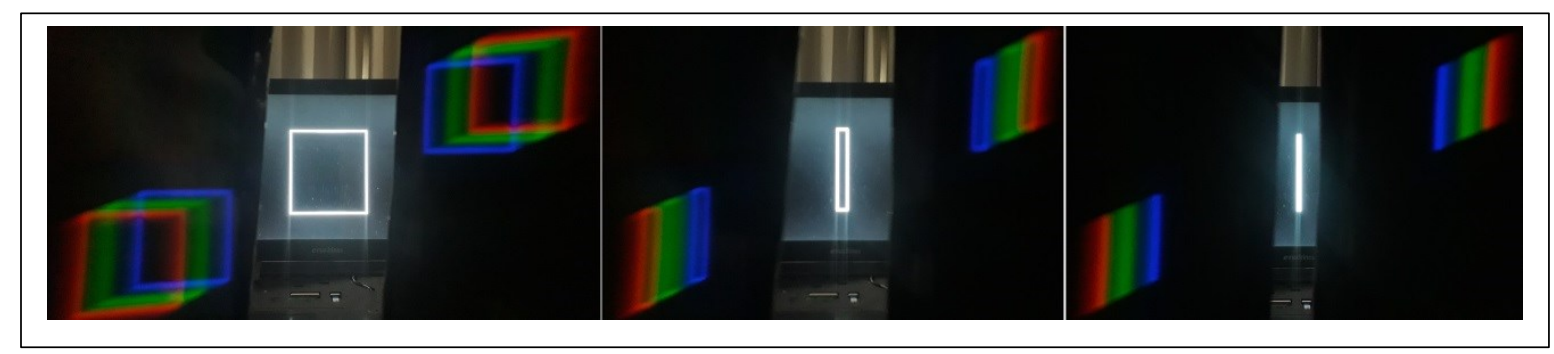

Fig. 4 - Imagen rectangular generada y proyectada por la laptop. El espectrómetro descompone la luz blanca de la laptop en sus tres frecuencias fundamentales según el sistema $R G B$ que utilizan las pantallas como televisores de plasma, monitores y lámparas LED.

En óptica se denomina colimar un haz de luz cuando se procede de manera que los rayos son paralelos entre sí (Hecht, 2000). Existen varias formas de lograr este objetivo, por ejemplo la luz proveniente de las estrellas ya se encuentra colimada debido a la gran distancia que se encuentra la fuente. La luz proveniente del Sol se encuentra colimada razonablemente, sus haces más extremos difieren en un ángulo de 30 minutos de arco. También podemos lograr el colimado utilizando lentes convergente, si la fuente se encuentra en el foco entonces 
los haces que salen de la lente se encuentran paralelos. El procedimiento realizado anteriormente de reducir el ancho del rectángulo (nuestra fuente) fue colimando los haces hasta permitir resolver las longitudes de onda de las componentes de la Laptop. Este procedimiento es muy habitual en espectroscopia utilizando estrechas rendijas que reducen el tamaño de la fuente.

\section{Resolución de líneas espectrales}

Cada red puede separar o resolver líneas espectrales próximas hasta un límite dado por la geometría misma de la red. Para continuar el análisis es necesario definir un parámetro denominado potencia dispersiva (D) (HECHT, 2000), para lo cual se utiliza la siguiente ecuación:

$$
\mathfrak{D}=\frac{\Delta \theta^{\prime} \text { (separacion angular entre lineas) }}{\Delta \lambda}=\frac{m}{a \cos \theta_{m}}
$$

Donde $\Delta \theta^{\prime}$ es la separación angular entre líneas, $\Delta \lambda$ es la diferencia entre dos longitudes de onda próximas y $m$ es el número de orden de los máximos, \pm 1 en este trabajo.

Retomando el ejemplo del doblete del mercurio y suponiendo que se desea conocer de antemano el tamaño de rendija a utilizar para lograr observar las dos líneas cuyas longitudes de ondas difieren en $\Delta \lambda=579 \mathrm{~nm}-577 \mathrm{~nm}=2 \mathrm{~nm}$. Sabiendo además que el CD ofrece una red de constante característica $a=1,6 \mu \mathrm{m}$ (KRUGLYAK, 1991; KNAUER, 2002), es posible obtener un valor de separación angular entre líneas para el caso del doblete de $0,0013 \mathrm{rad}$ o $1,3 \mathrm{mrad}$ (miliradianes). Cuando observamos un espectro discontinuo, la separación angular entre cada línea no puede ser menor que el ancho aparente de la rendija, por lo que:

$$
\frac{d}{L}<1,3 \operatorname{mrad}
$$

En la Fig. 5 se observa el doblete de mercurio utilizando una lámpara de vapor de mercurio para experiencias de laboratorio (PASCO Hg Vapor Light Source OS-9286). Se usó una rendija de $d=1,1 \mathrm{~mm}$ y se ubica nuestro espectrómetro a distintas distancias $L>0,85 \mathrm{~m}$. Obsérvese que se comienza a resolver el doblete recién a distancias superiores a 1 metro.

\section{EI desafío de observar las líneas de Fraunhofer}

Las llamadas líneas de Fraunhofer son líneas de absorción que se observan al ver el espectro solar. Esta absorción de las longitudes de onda se debe a las especies químicas que se encuentran en la fotosfera estelar y a los átomos dispersos en la atmósfera terrestre. Las líneas de absorción son problemáticas ya que si no se calibra correctamente el dispositivo (tamaño de rendija, distancia a la rendija o constante de la red) es probable que no se observe nada. Si 
el equipo no puede resolver un determinado ancho de línea espectral entonces la ausencia de luz que se desea ver queda solapada por la luz circundante.

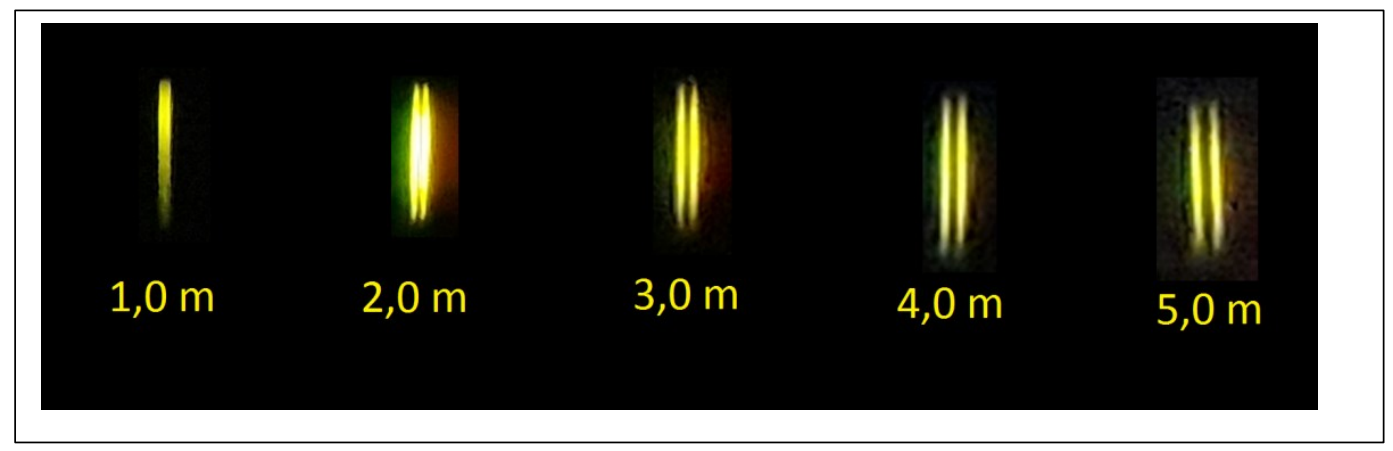

Fig. 5 - Fotografias ampliadas del doblete del mercurio obtenidos con el espectroscopio propuesta en este artículo. A medida que la cámara se va ubicando más lejos el doblete se va resolviendo, pero su fotografía se va achicando, cuestión que aqui no se observa ya que las imágenes han sido sucesivamente aumentando a través de la edición.

Las líneas, tanto sean de absorción o emisión, no son infinitamente delgadas. Esto se debe a la incertidumbre en la energía de transición atómica de los estados involucrados. El tiempo de duración de esta transición se encuentra entre $10^{-8}$ y $10^{-9}$ segundos (HAKEN; WOLF, 2000), lo que implica resolver un ancho de línea de $6 \times 10^{-15} \mathrm{~m}\left(6 \times 10^{-6} \mathrm{~nm}\right)$. Considerando que dicho valor es un resultado típico para las transiciones, esta incerteza es prácticamente imposible para el dispositivo propuesto. Sin embargo hay otros factores que aumentan el ancho de la línea, como ser el efecto Doppler de los átomos y moléculas a causa de su agitación térmica (ROHOLF, 1994). El movimiento térmico de los átomos que tiene velocidad dirigida hacia el observador tendrá frecuencias de emisión o absorción diferentes de las que presentarían si estuvieran en reposo. Por tal motivo, el efecto Doppler explica por qué las líneas de absorción que provienen del Sol tienen un ancho posible de ser observado por nuestro dispositivo. En efecto, ese ancho es determinante para programar de antemano que líneas van a poder ser observadas por el dispositivo propuesto.

A continuación, se presentan algunos valores de líneas espectrales del Sol, la tabla 1 fue armada en base a los datos aportados por distintas fuentes: Moore, Minnaert y Houtgast (1966), Luo y Gerritsen (1993), Lacarra y Majcher (2014) y Larson (2017).

En vista de que los anchos de las líneas de absorción tienen como cota máxima un valor igual al doblete del mercurio, se trabajó para el espectro solar con una rendija de aproximadamente la mitad de ancha que la usada para la Fig. 5 y a una distancia $L$ de aproximadamente 2 metros. 
Tabla 1 - Valores de líneas de absorción en el espectro solar de mayor ancho espectral.

\begin{tabular}{|c|c|c|c|}
\hline & $\begin{array}{c}\text { Longitud de onda } \\
(\mathbf{n m})\end{array}$ & $\begin{array}{c}\text { Ancho de la línea } \\
(\mathbf{n m})\end{array}$ & Origen \\
\hline $\mathbf{K}$ & 393,37 & 2,03 & $\mathrm{Ca}$ II \\
\hline $\mathbf{H}$ & 396,85 & 1,55 & $\mathrm{Ca} \mathrm{II}$ \\
\hline $\mathbf{G}$ & 434,05 & 0,29 & $\mathrm{H}(\gamma)$ \\
\hline $\mathbf{F}$ & 486,13 & 0,37 & $\mathrm{H}(\beta)$ \\
\hline $\mathbf{b}$ & 518,36 & 0,19 & $\mathrm{Mg} \mathrm{I}$ \\
\hline $\mathbf{E}$ & 526,96 & 0,05 & $\mathrm{Fe} \mathrm{I}$ \\
\hline $\mathbf{D}_{\mathbf{2}}$ & 589,00 & 0,08 & $\mathrm{Na} \mathrm{I}$ \\
\hline $\mathbf{D}_{\mathbf{1}}$ & 589,59 & 0,06 & $\mathrm{Na} \mathrm{I}$ \\
\hline $\mathbf{C}$ & 656,28 & 0,40 & $\mathrm{H}(\alpha)$ \\
\hline B & 686,99 & 0,04 & Oxigeno $($ terrestre $)$ \\
\hline
\end{tabular}

\section{Procedimientos prácticos}

Los materiales necesarios para realizar la experiencia son: una lámpara LFC, un espejo común, una rendija, el sector circular de CD "depilado" y un teléfono smartphone. Además, es de gran utilidad contar con un soporte para el teléfono y otro soporte regulable para el espejo. Para la lámpara LFC se necesita contar con un portalámparas correctamente aislado y maniobrable. Cartones y cartulinas color negro opaco son de gran utilidad para limitar los reflejos al momento de sacar las fotos

La rendija se puede construir a partir de una hoja de afeitar seccionada a la mitad y al acercarlas por el filo se conformará una rendija de gran precisión. En caso de no conseguir hojas de afeitar se puede utilizar papel de aluminio cortado con una cuchilla afilada. La separación de la rendija utilizada para la observación de las líneas de Fraunhofer fue de 0,7 $\mathrm{mm}$. Esta distancia se puede estimar haciendo incidir un haz de láser sobre la rendija y posteriormente medir el ancho del lóbulo central de difracción que produce sobre una pantalla. Procedimiento muy conocido en la física experimental de óptica (HECHT, 2000).

Antes de comenzar el procedimiento es conveniente advertir que se debe evitar en todo momento la observación directa a ojo desnudo de los haces de luz provenientes del Sol, ya sea directamente o a través de la rendija. Recomendamos también evitar que la cámara apunte directamente al Sol durante largo períodos para evitar daños irreversibles. En esta práctica la intensidad de los rayos solares que lleguen a la cámara del smartphone se verán reducidos en intensidad al pasar antes por una rendija. El procedimiento que describiremos a continuación se basa en el hecho que una fotografía de un espectro no provee ninguna información útil a menos que podamos tener otro espectro de referencia del cual se conozca la posición de las líneas de referencia y longitud de onda correspondiente. En este caso para 
poder facilitar la tarea del docente que quiera replicar estos resultados con sus estudiantes, se propone hacer uso de una LFC. Dichas lámparas presentan una serie de líneas de emisión características del mercurio a baja presión y del recubrimiento que tiene en su interior el tubo. Los valores de estas líneas fueron medidas con un espectrómetro recientemente desarrollado por el Grupo de Investigación "Docencia Experimental de la Física" del Departamento de Física de la Facultad de Ciencias Exactas y Naturales de la Universidad Nacional de Mar del Plata, (SZIGETY; BERNAL; BILBAO, 2016). El espectro observado para la LFC se muestra en el Gráfico 1.

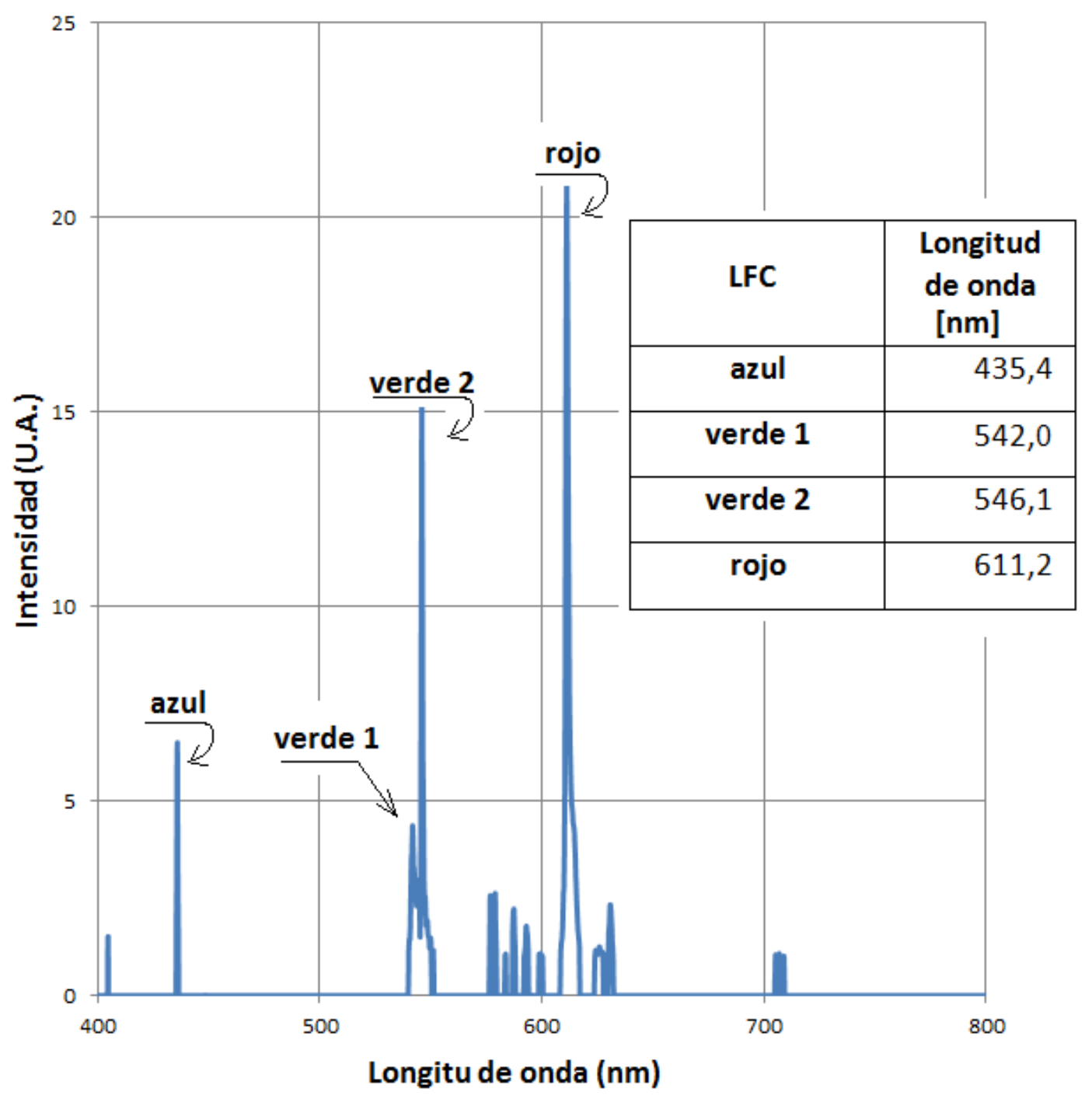

Gráfico 1 - Espectro de emisión para una lámpara fluorescente compacta (LFC).

Se deben tomar dos fotografías con el teléfono, y para que de ambas se puedan obtener resultados coherentes, la distancia entre rendija y la cámara debe mantenerse inalterable, así como la posición de la cámara. 
El arreglo experimental sugerido se observa en la Fig. 6. La Fig. 6-A muestra la disposición para el caso de rayo de luz solar y la Fig. 6-B el armado para la lámpara. Se puede buscar una habitación oscurecida, aunque no es condición necesaria que esté completamente oscura. Debe considerarse que los reflejos de luz de objetos iluminados por el Sol en la habitación pueden afectar la medición, por lo que se sugiere realizarlos en un salón con luz de atardecer.

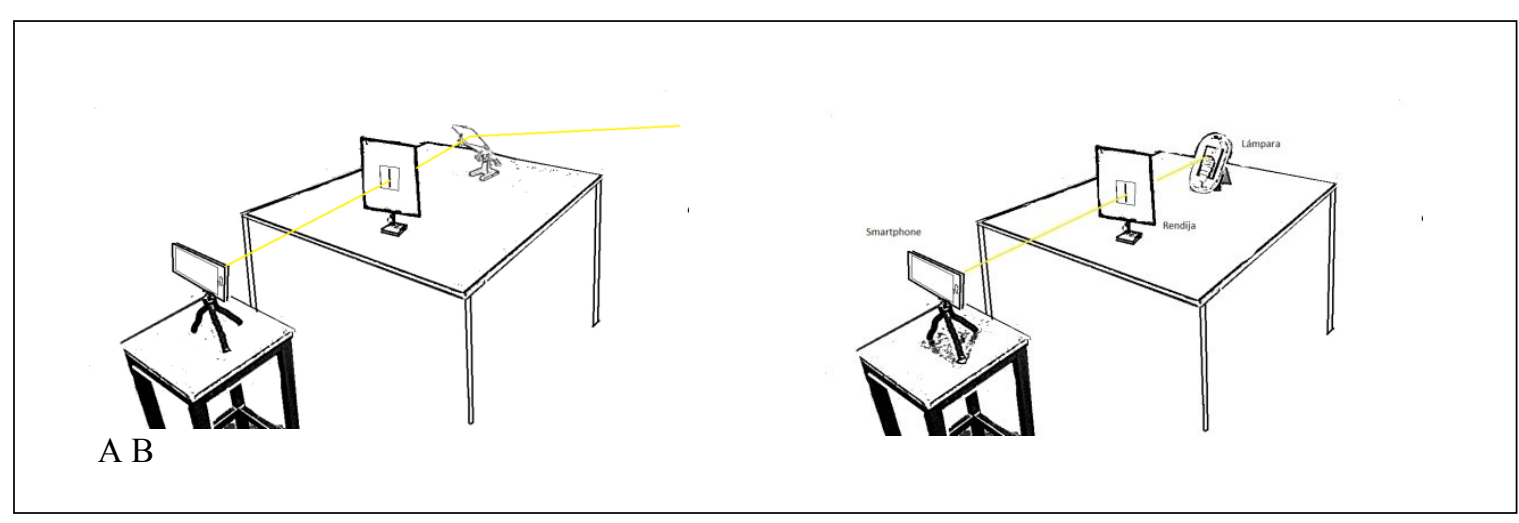

Fig. 6 - Arreglo experimental. Disposición de la fuente de luz (espejo o lámpara LFC), rendija con un marco de cartón y teléfono. Todo montado en una misma línea.

A continuación, se propone una serie de pasos que permitirán mejorar los resultados.

$1^{\circ}$ Paso) Permitir que en la habitación ingrese un rayo de luz solar. Utilizar un espejo con su soporte y ubicarlos hasta que un reflejo del Sol se proyecte en la pared de la habitación. Tenga cuidado con este reflejo y evite que incida sobre los ojos de los estudiantes que estén realizando la experiencia.

$2^{\circ}$ Paso) Ubicar la cámara a una distancia del espejo. Y luego mover el espejo para que el reflejo incida en la lente de la cámara

$3^{\circ}$ Paso) Interponer la rendija entre el haz que refleja el espejo y la cámara. Ajustar su altura para que, desde la cámara, se pueda ver la rendija iluminada. Esta distancia es la que importa en los cálculos y en este caso es $L=2,12+/-0.01 \mathrm{~m}$

$4^{\circ}$ Paso) Se puede utilizar una cartulina negra opaca de tal forma que al momento de sacar la fotografía del espectro se eliminen reflejos indeseados y permitir un fondo negro para que el espectro sea nítido (ver Fig. 7). Una vez que se observe en la pantalla del teléfono la imagen deseada del espectro del Sol, tomar la foto utilizando el temporizador para evitando cualquier vibración. Esta función está disponible en la mayoría de las cámaras y permite sacar la foto unos segundos después de apretar el obturador. Se puede observar en la figura 9 los dos espectros que se forman a ambos costados de la imagen de la rendija.

$5^{\circ}$ Paso) Tomar la segunda foto sin mover la cámara ni la rendija. Esta vez se obtendrá el espectro de referencia de la LFC. Para ello retirar el espejo y ubicar la lámpara detrás de la rendija. 
Recomendación: al momento de tomar la foto fijar el foco de la cámara y bajar la intensidad de luz que recibe, probar esto con distintos modelos de ajuste que suelen traer las aplicaciones de las cámaras.

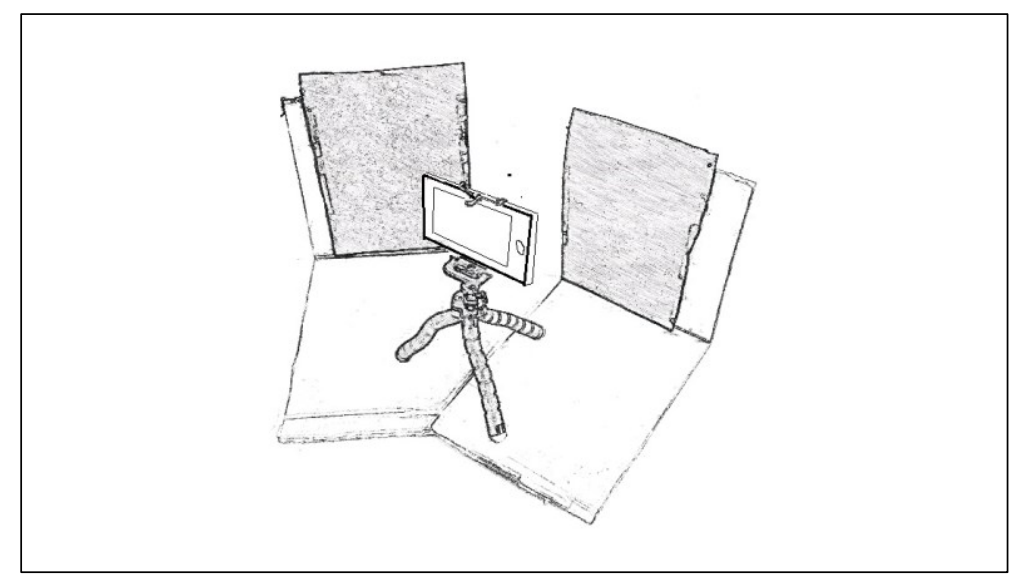

Fig. 7 - Cartulinas oscuras en soportes " $L$ ” de cartón para tener un fondo negro en la visualización del espectro en caso de algún reflejo de la habitación moleste en la fotografia final suficientemente oscura.

\section{Resultados obtenidos}

Programas como el Paint u cualquier otro que permita visualizar la ubicación del cursor pixel a pixel permiten obtener la ubicación angular de las líneas sobre la foto. En la Fig. 8 se observa la pantalla del Paint y la ubicación de la información necesaria para poder realizar mediciones sobre la foto digital.

Cualquier objeto de la fotografía puede ser medido para poder tener una referencia de escalas. Debido a que ya tenemos la distancia $L$ medida con anterioridad usaremos como objeto de referencia el cartón que conforma la cubierta de la rendija. El ancho del mismo es de $X=21,0+/-0,5 \mathrm{~cm}$. Al abrir la foto en el Paint, se traza una línea sobre el alto de la cubierta y la misma resulta de $X^{`}=322,0+/-0,5$ pixel. La proporción de escala entre estas dos medidas $\left(e=X / X^{\prime}\right)$ es la que permite convertir la ubicación de las líneas del espectro en la foto en posiciones angulares. Si la posición de una línea tiene componentes en $x^{`}$ e $y^{\prime}$ en pixeles, se realiza el cálculo de la hipotenusa con el teorema de Pitágoras para obtener $X_{\text {linea }}$ de cualquier posición sobre la fotografía.

Para obtener la ubicación angular $\theta_{m}$ tenemos que resolver el triángulo rectángulo formado entre la rendija, la cámara del teléfono y la ubicación de la línea. Para eso debemos disponer de la distancia $(L)$ entre la cámara y la rendija.

$$
\theta_{m}=\operatorname{arctg}\left(X_{\text {linea }} \cdot e / L\right)
$$




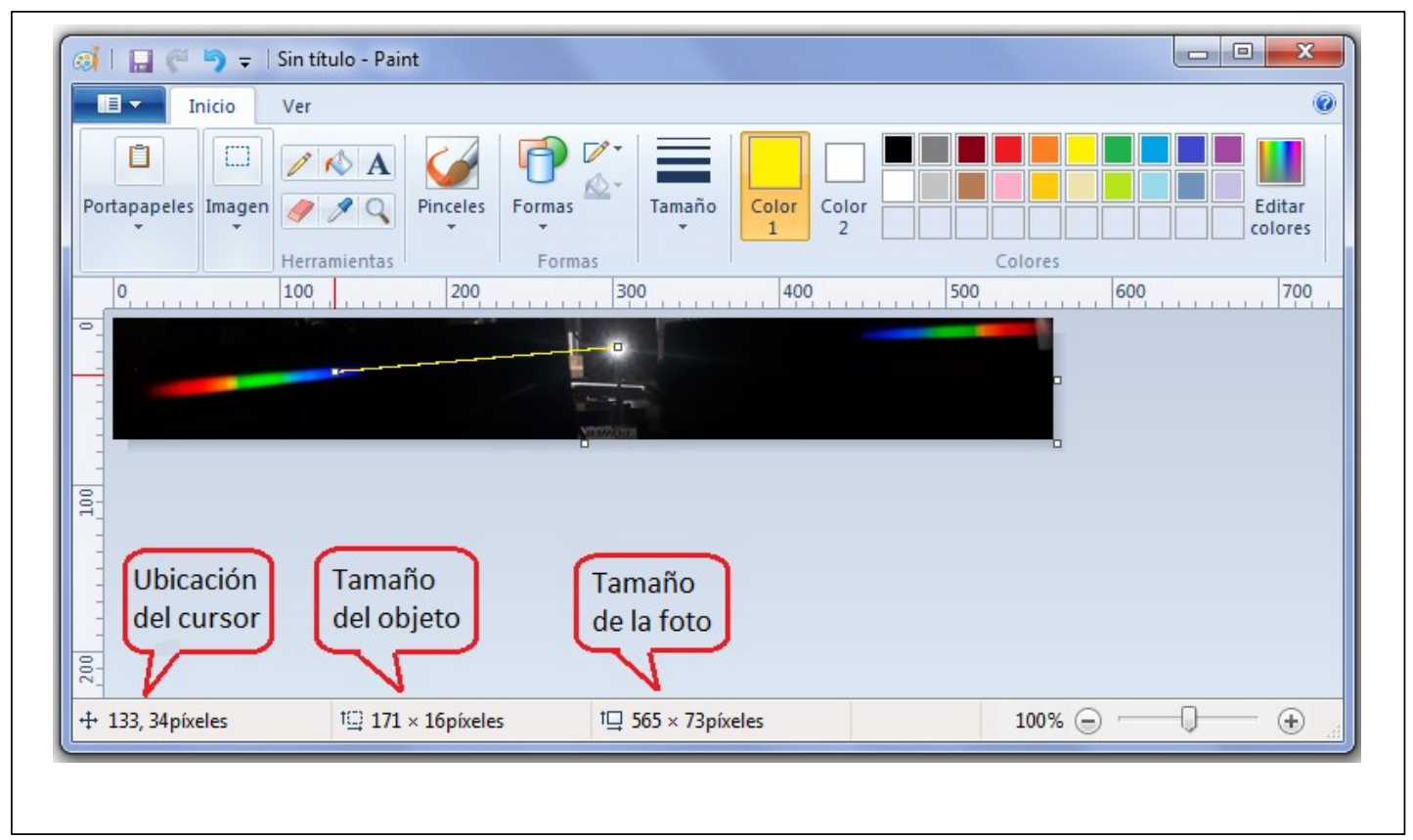

Fig. 8 - En la barra de estado de Paint se encuentra el tamaño del objeto creado, en este caso la línea amarilla que va desde la rendija a las líneas de absorción que se desea medir.

En base esta expresión podemos comenzar a identificar las líneas de la lámpara LFC. En la Tabla 2 se puede observar el resultado obtenidos para la LFC en base a la foto de la Fig. 9 y la grafica 1. En base a estos datos podemos calibrar las distancias con las longitudes de onda en la fotografía del espectro solar.

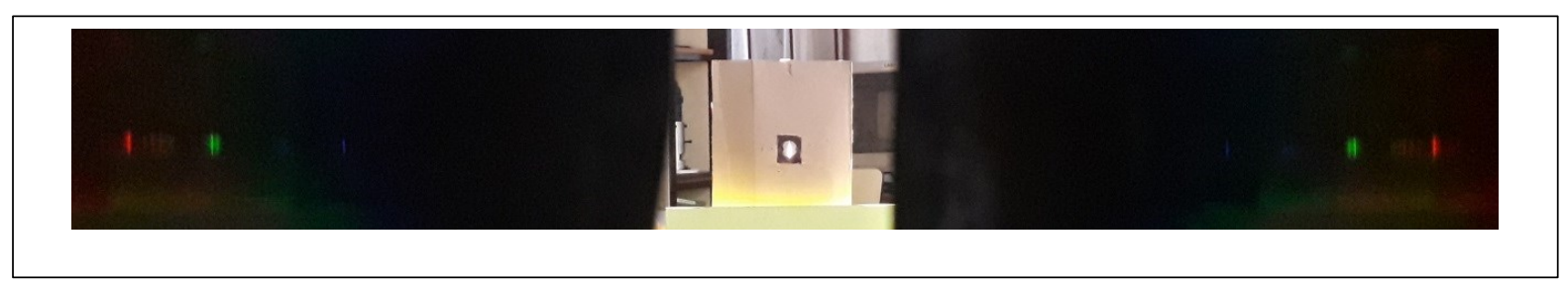

Fig. 9 - Foto en base a la cual se obtuvieron los datos del espectro de la LFC. La línea de emisión del azul resulta un tanto tenue pero se puede localizar y medir su posición. 
Tabla 2 - Datos obtenidos de la fotografía de la Fig. 9 para el espectro de la Izquierda (Izqu) y derecha (Der).

\begin{tabular}{|l|c|c|c|c|c|}
\hline \multicolumn{1}{|c|}{$\begin{array}{c}\text { Líneas }_{\text {de la LFC }} \\
\text { [pixel] } \\
+/-0,5\end{array}$} & $\begin{array}{c}y^{\prime} \\
\text { [pixel] } \\
+/-0,5\end{array}$ & $\begin{array}{c}\text { Módulo } \\
\left(X^{\prime} \text { linea }\right. \\
{[\text { pixel] }} \\
+/-0.5\end{array}$ & $\begin{array}{c}\text { Ángulo }\left(\theta_{m}\right) \\
{[\mathrm{rad}]} \\
+/-0.01\end{array}$ & $\begin{array}{c}\text { Orden } \times \text { Longitud de } \\
\text { onda referencia } \\
(m)\end{array}$ \\
\hline $\begin{array}{l}\text { Azul } \\
\text { (Izqu) }\end{array}$ & 1022 & 7 & 1022,0 & 0,30 & $+\mathrm{nm}]$ \\
\hline $\begin{array}{l}\text { Verde 1 } \\
\text { (Izqu) }\end{array}$ & 1315 & 11 & $1315,0.1$ \\
\hline $\begin{array}{l}\text { Verde 2 } \\
\text { (Izqu) }\end{array}$ & 1327 & 11 & 1327,0 & 0,38 & 435,4 \\
\hline $\begin{array}{l}\text { Rojo } \\
\text { (Izqu) }\end{array}$ & 1517 & 15 & 1517,1 & 0,43 & 542,0 \\
\hline $\begin{array}{l}\text { Azul } \\
\text { (Der) }\end{array}$ & 1003 & 6 & $-1003,0$ & $-0,30$ & 611,2 \\
\hline $\begin{array}{l}\text { Verde 1 } \\
\text { (Der) }\end{array}$ & 1286 & 5 & $-1286,0$ & $-0,37$ & $-435,4$ \\
\hline $\begin{array}{l}\text { Verde 2 } \\
\text { (Der) }\end{array}$ & 1299 & 6 & $-1299,0$ & $-0,38$ & -542 \\
\hline $\begin{array}{l}\text { Rojo } \\
\text { (Der) }\end{array}$ & 1481 & 8 & $-1481,0$ & $-0,42$ & $-546,1$ \\
\hline
\end{tabular}

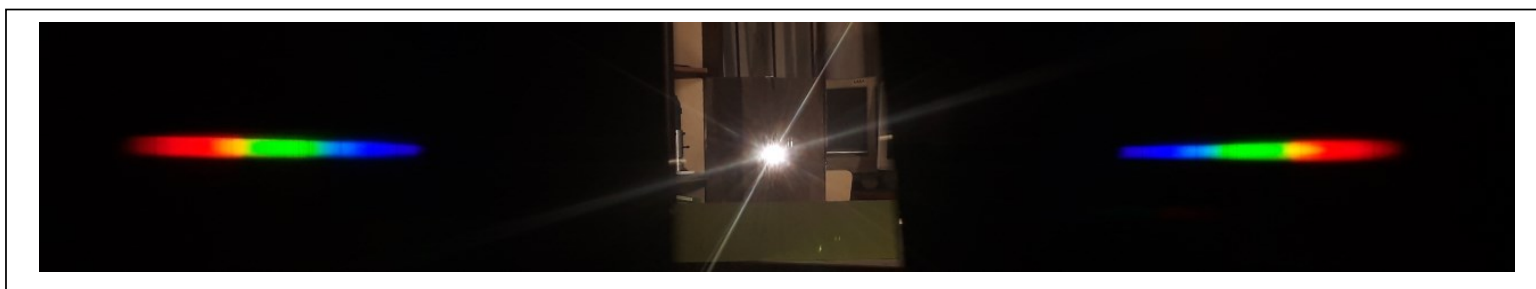

Fig. 10 - Foto en base a la cual se obtuvieron los datos del espectro del Sol.

Tabla 3 - Datos obtenidos para el espectro solar Sol.

\begin{tabular}{|c|c|c|c|c|}
\hline $\begin{array}{c}\text { Líneas de } \\
\text { absorción } \\
\text { contando } \\
\text { desde el azul }\end{array}$ & $\begin{array}{c}x^{\prime} \\
\text { [pixel] } \\
+/-0,5\end{array}$ & $\begin{array}{c}y^{\prime} \text { [pixel] } \\
+/-0,5\end{array}$ & $\begin{array}{c}\left.\text { Módulo }\left(X_{\text {linea }}^{\prime}\right)\right) \\
{[\text { pixel] }} \\
+/-0.5\end{array}$ & $\begin{array}{r}\text { Ángulo }\left(\theta_{m}\right) \\
{[\mathrm{rad}]} \\
+/-0.01\end{array}$ \\
\hline $1^{\text {o }}$ & 1010 & 15 & 1010,1 & 0,30 \\
\hline $2^{\text {o }}$ & 1158 & 13 & 1158,1 & 0,34 \\
\hline $3^{\text {o }}$ & 1244 & 15 & 1244,1 & 0,36 \\
\hline $4^{\text {o }}$ & 1272 & 13 & 1272,1 & 0,37 \\
\hline
\end{tabular}




\begin{tabular}{|c|c|c|c|c|}
\hline $5^{\mathbf{0}}$ & 1449 & 20 & 1449,1 & 0,42 \\
\hline $6^{\mathbf{o}}$ & 1653 & 26 & 1653,2 & 0,47 \\
\hline $1^{\mathbf{o}}$ & 993 & 9 & 993,0 & 0,29 \\
\hline $2^{\mathbf{o}}$ & 1137 & 7 & 1137,0 & 0,33 \\
\hline $3^{\mathbf{o}}$ & 1218 & 10 & 1218,0 & 0,36 \\
\hline $4^{\mathbf{o}}$ & 1244 & 9 & 1244,0 & 0,36 \\
\hline $5^{\mathbf{o}}$ & 1417 & 9 & 1417,0 & 0,41 \\
\hline $6^{\mathbf{o}}$ & 1612 & 10 & 1612,0 & 0,46 \\
\hline
\end{tabular}

\section{Análisis de los datos}

Utilizando los datos de las dos últimas columnas de la tabla 2, se grafica el seno del ángulo $\theta_{m}$ en función de la longitud de onda de la lámpara de LFC. La grafica 2 es el resultado de esta correlación con la recta de mejor ajuste máxima y mínima que servirá como ecuación de calibración para las medidas que tomaremos del Sol. La clara linealidad de esta relación de puntos confirma la expresión (1) para los órdenes 1 y -1.

La pendiente de la recta de calibración nos da una medida de la constante $a$ en nanómetros del CD y su ordenada al origen nos indica que la perpendicularidad entre la red y el haz incidente no es perfecta, existe un ángulo $\theta_{i}$. Los problemas de alineación del arreglo experimental no acarrean problemas si las dos fotos son obtenidas en la misma posición de la cámara, es decir que no se ha movido la cámara ni la red. Solo se cambia la fuente que ilumina la rendija.

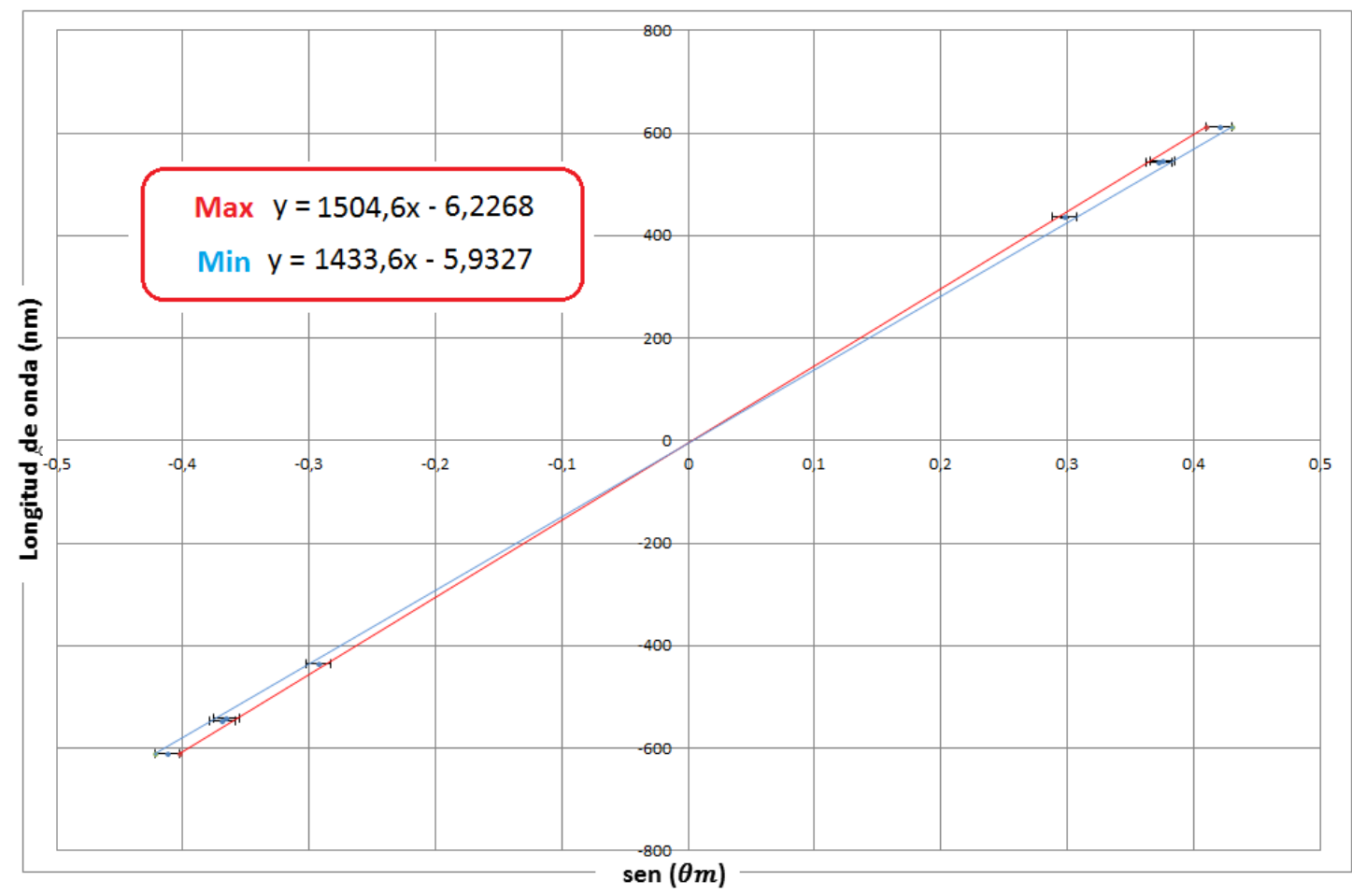

Grafica 2 - Ecuación de calibración. Seno (ángulo máx. orden =1 y -1) vs longitud de onda teórica en $[\mathrm{nm}]$. 
Finalmente, por medio de la ecuación de calibración es posible convertir los valores de ángulo de la tabla 3 en su equivalente de longitud de onda. La pendiente de la ecuación de calibración y su ordenada al origen se obtuvieron con un error en base a la pendiente máxima y mínima de la Grafica 2. La pendiente de calibración resultó 1470 +/- $40 \mathrm{~nm}$ y la ordenada al origen de calibración $6,08+/-0,15 \mathrm{~nm}$.

Especializando los ángulos $\theta_{m}$ de la tabla 3 en la ecuación de calibración se obtiene las longitudes de onda de las líneas de absorción. Los resultados se presentan en la tabla 4 con su correspondiente incerteza, la identificación de la línea y error porcentual.

Tabla 4 - Resultado y márgenes de error de las líneas reconocidas en la fotografía.

\begin{tabular}{|c|c|c|c|}
\hline $\begin{array}{c}\text { Longitud de onda } \\
\text { medida (nm) }\end{array}$ & Incerteza & $\begin{array}{c}\text { Referencia teórica de las } \\
\text { líneas (nm) }\end{array}$ & $\begin{array}{c}\text { Error porcentual } \\
\mathbf{( \% )}\end{array}$ \\
\hline 430 & $+/-20$ & Línea G $(434,05)$ & 1,5 \\
\hline 480 & $+/-30$ & Línea F $(486,13)$ & 0,39 \\
\hline 520 & $+/-30$ & Línea b $(518,36)$ & 0,43 \\
\hline 530 & $+/-30$ & Línea E $(526,96)$ & 0,13 \\
\hline 590 & $+/-30$ & Línea D doblete & 0,19 \\
\hline 660 & $+/-30$ & Línea C $(6569,28)$ & 0,077 \\
\hline-430 & $+/-20$ & Línea G $(434,05)$ & 0,29 \\
\hline-490 & $+/-30$ & Línea F (486,13) & 0,48 \\
\hline-520 & $+/-30$ & Línea b (518,36) & 0,066 \\
\hline-530 & $+/-30$ & Línea E $(526,96)$ & 0,23 \\
\hline-590 & $+/-30$ & Línea D doblete & 0,0062 \\
\hline-650 & $+/-30$ & Línea C $(656,28)$ & 0,089 \\
\hline
\end{tabular}

En la Fig. 11 se observa un detalle aumentado de la foto de la Fig. 10. Los dos espectros obtenidos, uno de ellos invertido con un programa gráfico y reducido su brillo y contraste para resaltar las líneas de absorción. En base a los datos de las fuentes antes mencionadas se identificaron las líneas más anchas, las llamadas C, el doblete D, E, F, G y b. 


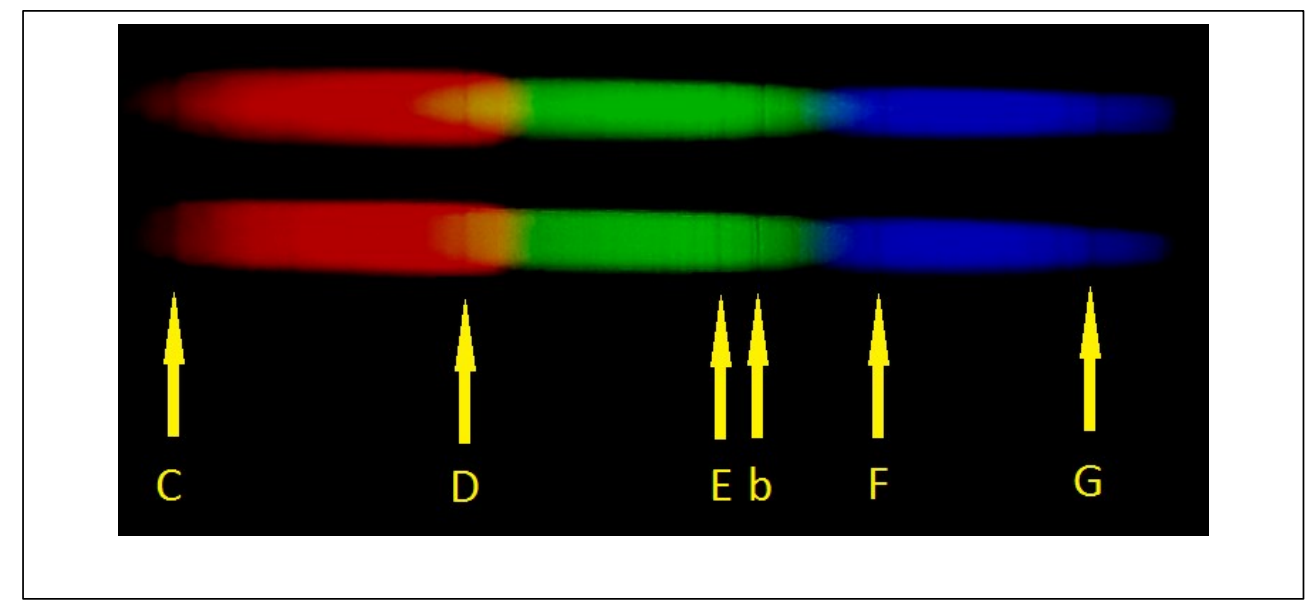

Fig. 11 - Detalle ampliado de las líneas espectrales de mayor ancho observado. La primera contando de arriba es el espectro obtenido a la derecha de la Fig. 9 (claramente invertido). El segundo corresponde a la izquierda.

\section{Conclusiones}

Las mediciones de las longitudes de onda de las líneas de absorción respecto de los valores de tabla tienen un error porcentual máximo de 1,5\%, mientras que la precisión de los valores tiene una incerteza relativa del 5\%. En base a estas consideraciones podemos decir que los procedimientos propuestos en este artículo pueden ser mejorados pero cabe menciona que dichos márgenes de exactitud y precisión se consideran suficientes para un trabajo práctico de laboratorio con fines didácticos. También hay que considerar que el dispositivo central es el uso de la cámara de un teléfono de gama media con una resolución de $13 \mathrm{Mpx}$ (Mega pixeles) y un CD grabable de uso corriente. Bajo este contexto concluimos que la propuesta es una práctica de bajo costo que permite realizar una actividad didáctica de la física experimental relacionada con la fisca ondulatoria y astronómica.

A pesar de que el procedimiento propuesto puede ser complicado para la enseñanza de la física en escuelas secundaria, para estudiantes universitarios resulta una práctica introductoria a las actividades experimentales ya que hay que hacer uso de ajuste de recta por mínimos cuadrados, propagación de errores y procesamiento sencillo de imágenes digitales. Los autores actualmente se encuentran explorando distintas propuestas didácticas que puedan servir para fortalecer contenidos conceptuales para distintas temáticas de la física donde se encuentren involucrada el análisis de espectros de emisión y de absorción.

Para alumnos de instituciones de enseñanza media, los autores consideran que este dispositivo puede permitir a los docentes de física despertar el interés de los estudiantes por 
los análisis espectrales y la descomposición de la luz. Invitamos a la comunidad a proponer actividades para ser desarrolladas con esta técnica donde estudiantes puedan observar y analizar en forma cualitativa y cuantitativa distintos espectros de emisión y absorción. La oportunidad que ofrece este espectroscopio casero es que el adolescente encuentre en su teléfono o smartphone una herramienta para analizar y comprender el mundo natural y físico. Pudiendo generar algún debate epistemológico sobre lo que el científico detecta del mundo cuando utiliza un instrumento para verlo, entre otros análisis que se enmarquen dentro del contexto de descubrimiento científico.

Una última consideración acerca de esta práctica es proponer alguna mejora. Por ejemplo, se puede intentar cambiar el CD por un DVD el cual tiene un espaciado $a$, menor de líneas. Lo cual mejoraría el poder de resolución de la red. Esto trae aparejado la depilación de estos discos los cuales tiene una estructura de capas más compleja que el CD común. El CD ya de por si presenta la posibilidad de disponer de una red de gran poder resolutivo, a pesar de lo que se ha comentado en algun artículo sobre su incapacidad para resolver el doblete del mercurio (WAKABAYASHI; HAMADA, 2006). Este espectroscopio tiene un costo bajo y versatilidad muy alta por lo que todavía no se ha explorado todo lo que se puede hacer con él tanto en versión fotográfica como en su versión de video para obtener espectros variables en el tiempo.

\section{Bibliografía}

BURMAN, G. A. Overhead spectroscopy. The Physics Teacher, v. 29, n. 7, p. 470-470, oct 1991.

CATELLI, F. Demonstre em aula: projeção de espectros com um cd e retroprogetor. Caderno Brasileiro de Ensino de Física, v. 16, n. 1, p. 123-126, abr. 1999.

CATELli, F.; HERBER, J.; MENEGOTTO, J.; KESSLER, S. O que é uma raia espectral. A Física na Escola, v. 7, n. 2, p. 70-72, 2006.

CATELli, F.; GIOVANNINI, O.; DE OLIVEIRA, S. F. Espectrômetro amador: quantificando comprimentos de onda. Caderno Brasileiro de Ensino de Física, v. 34, n. 3, p. 951-970, dez. 2017.

HAKEN, H.; WOLF, H. C. The physics of atoms and quanta: introduction to experiments and theory. 7. ed. Berlin: Springer Science \& Business Media, 2005.

HECHT, E. Óptica. 3. ed. Madrid: Addisson Wesley Iberoamericana, 2000. 
HOWE, J.; SITAR, D. J. Using RSpec in an introductory bright star spectroscopy lab activity. The Physics Teacher, v. 56, n. 1, p. 56-57, 2018.

KALINOWSKI, H. J.; GARCIA, N. M. D. Laboratório caseiro: Uma alternativa econômica para redes de difração no laboratório de ensino. Caderno Brasileiro de Ensino de Física, v. 7, n. 1, p. 64-72, 1990.

KNAUER, T. A compact disk transmission spectroscope. The Physics Teacher, v. 40, n. 8, p. 466-467, 2002.

KRUGLYAK, H. The compact disc as a diffraction grating. Physics Education, v. 26, n. 4, p. 255-255, 1991.

LACARRA, M.; MAJCHER, A. Observations of various spectra with a home-made spectroscope, 2014. Madrid. Disponível em: <http://www.euhou.net/index.php/exercisesmainmenu-13/classroom-experiments-and-activities-mainmenu-186/179-observations-ofvarious-spectra-with-a-home-made-spectroscope>. Acesso em: 07 nov. 2019.

LARSON, A. Identifying Elements in the Sun Using Spectral Lines, 2017. New York. Disponível em: <http://www.columbia.edu/ vjd1/Solar\%20Spectrum\%20Ex.html>. Acesso em: 16 jul. 2019.

LINCOLN, J. Video spectroscopy with the RSpec Explorer. The Physics Teacher, v. 56, n. 3, p. 188-189, 2018.

LUO, W. W; GERRISTSEN, H. J. Seeing the Fraunhofer lines with only a diffraction grating and a slit. American journal of physics, v. 61, n. 7, p. 632-636, 1993.

MOORE, C. E.; HOUTGAST, J.; MINNAERT, M. G. J. The Solar Spectrum 2935 a to 8770 a: 2nd Revision of Rowland's Preliminary Table of Solar Spectrum Wavelength. Washington: US Government Printing Office, 1966.

ROHLF, J. W. Modern Physics from $\boldsymbol{\alpha}$ to $Z^{\mathbf{0}}$. 1. ed. New York: Wiley-VCH, 1994.

SADLER, P. Projecting spectra for classroom investigations. The Physics Teacher, v. 29, n. 7, p. 423-427, 1991.

SZIGETY, E. G.; BERNAL, L. J.; BILBAO, L. Propuesta de un trabajo práctico de laboratorio sobre corrimiento Doppler óptico en el aula universitaria. Revista Eureka sobre Enseñanza y Divulgación de las Ciencias, v. 14, n. 1, p. 135-143, 2016. 
WAKABAYASHI, F.; HAMADA, K. A DVD spectroscope: A simple, high-resolution classroom spectroscope. Journal of Chemical Education, v. 83, n. 1, p. 56-56, 2006.

WAKABAYASHI, F.; HAMADA, K.; SONE, K. CD-ROM spectroscope: A simple and inexpensive tool for classroom demonstrations on chemical spectroscopy. Journal of chemical education, v. 75, n. 12, 1998.

ZANETTI, V.; HARRIS, J. Spectra of Three Light Sources with a CD. Physics Teacher, v. 31, n. 2, 1993. 